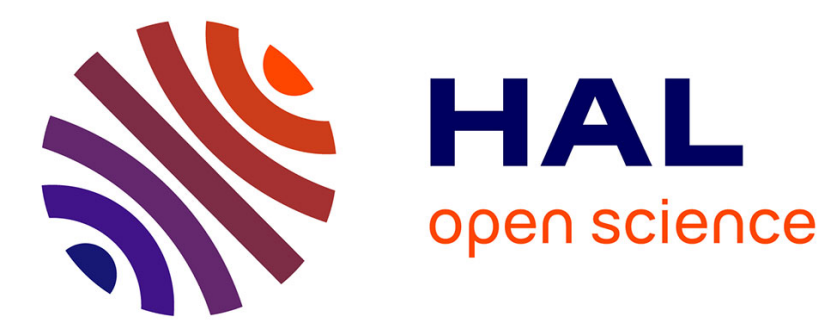

\title{
Galectin-3: A key player in arthritis
}

Yong Hu, Mélissa Yéléhé-Okouma, Hang-Korng Ea, Jean-Yves Jouzeau, Pascal Reboul

\section{To cite this version:}

Yong Hu, Mélissa Yéléhé-Okouma, Hang-Korng Ea, Jean-Yves Jouzeau, Pascal Reboul. Galectin-3: A key player in arthritis. Joint Bone Spine, 2017, 84 (1), pp.15-20. 10.1016/j.jbspin.2016.02.029 . hal-01704704

\section{HAL Id: hal-01704704 \\ https://hal.univ-lorraine.fr/hal-01704704}

Submitted on 8 Feb 2022

HAL is a multi-disciplinary open access archive for the deposit and dissemination of scientific research documents, whether they are published or not. The documents may come from teaching and research institutions in France or abroad, or from public or private research centers.
L'archive ouverte pluridisciplinaire HAL, est destinée au dépôt et à la diffusion de documents scientifiques de niveau recherche, publiés ou non, émanant des établissements d'enseignement et de recherche français ou étrangers, des laboratoires publics ou privés.

\section{(이) $\$$}

Distributed under a Creative Commons Attribution - NonCommercial - NoDerivatives| 4.0 


\section{Galectin-3: a key player in arthritis}

Y. Hu ${ }^{1,2}$, M. Yéléhé-Okouma ${ }^{1,3}$, HK. Ea ${ }^{4}$, JY. Jouzeau ${ }^{1,3}$, P. Reboul ${ }^{1}$

Yong $\mathrm{Hu}, \mathrm{MD}, \mathrm{PhD}$

Mélissa Yéléhé-Okouma, PharmD, PhD

Hang-Korng Ea, MD, PhD

Jean-Yves Jouzeau, PharmD, PhD

Pascal Reboul, $\mathrm{PhD}$

${ }^{1}$ UMR 7365 CNRS-Université de Lorraine IMoPA, France

${ }^{2}$ Department of orthopedics, Renmin Hospital, Wuhan University, Wuhan, China

${ }^{3}$ Centre Hospitalier Régional Universitaire, Nancy, France

${ }^{4}$ AP-HP, hôpital Lariboisière, Service de rhumatologie (centre Viggo Petersen), Pôle appareil locomoteur; Sorbonne Paris Cité, Université Paris Diderot; INSERM UMR-S 1132 Bioscar, France.

\section{Address correspondence to:}

Pascal Reboul, PhD, UMR 7365 CNRS-Université de Lorraine, IMoPA, Biopôle de l'Université de Lorraine, Campus Biologie-Santé, Faculté de Médecine, bureau 2G66, 9 avenue de la forêt de Haye- CS50184, 54505 Vandœuvre lès Nancy Cedex.

Tel: +33 3836854 33, Fax: +33 383685409

Email: pascal.reboul@univ-lorraine.fr 


\begin{abstract}
Arthritis is more and more considered as the leading reason for the disability in the world, particularly regarding its main entities, rheumatoid arthritis and osteoarthritis. The common feature of arthritis is inflammation, which is mainly supported by synovitis (synovial inflammation), although the immune system plays a primary role in rheumatoid arthritis and a secondary one in osteoarthritis. During the inflammatory phase of arthritis, many pro-inflammatory cytokines and mediators are secreted by infiltrating immune and resident joint cells, which are responsible for cartilage degradation and excessive bone remodeling. Amongst them, a $\beta$-galactoside-binding lectin, galectin-3, has been reported to be highly expressed and secreted by inflamed synovium of rheumatoid arthritis and osteoarthritis patients. Furthermore, galectin-3 has been demonstrated to induce joint swelling and osteoarthritis-like lesions after intra-articular injection in laboratory animals. However, the mechanisms underlying its pathophysiological role in arthritis have not been fully elucidated. This review deals with the characterization of arthritis features and galectin-3 and summarizes our current knowledge of the contribution of galectin-3 to joint tissue lesions in arthritis.
\end{abstract}

Key words: galectin-3, rheumatoid arthritis, osteoarthritis, synovitis, cartilage, bone. 


\section{Introduction}

Arthritis is mainly composed of rheumatoid arthritis (RA) and osteoarthritis (OA). Pain, inflammation and stiffness of the joints are the main common symptoms leading to disability, whereas bone changes vary from subchondral sclerosis and osteophytosis in OA to erosions and osteoporosis in RA. In France the prevalence of radiographic OA was estimated of about $17 \%$ and a recent study indicates that the prevalence of symptomatic knee OA is around $2 \%$ for both males and females of 40 to 49 years-old, while increasing to 10 to $15 \%$ for people between the age of 70 to 75 years [1]. In contrast, the RA prevalence is $0.3 \%$ in France [2].

The aetiology of each disease is different, as RA is an autoimmune inflammatory disease while $\mathrm{OA}$ is generally considered as a degenerative/mechanical one. In addition, genetic and environmental influences do not overlap between diseases, while autoantibody production is restricted to RA. However, inflammation of the synovial membrane is a prominent feature of OA pathogenesis that results in synovitis, which is thought to reflect structural progression of OA [3]. Synovial inflammation is a primary event in RA whereas it can be an early, as illustrated by tissue thickening detected by MRI [4] or a late event, secondary to cartilage breakdown, in OA. Although synovitis is frequent in OA, the level of inflammation remains lower than in RA and pannus formation occurs scarcely, whereas it is a major characteristic of RA [5]. Such grading of inflammation comes from the much lower contribution of cells of the innate (mainly phagocytes) and adaptative (B and $\mathrm{T}$ lymphocytes) immune responses in OA than in RA. Synovitis induces cartilage destruction and accounts for bone erosion in RA while promoting subchondral bone (SCB) remodeling in OA. Within the pannus, where leukocyte infiltration occurs beside proliferation of the

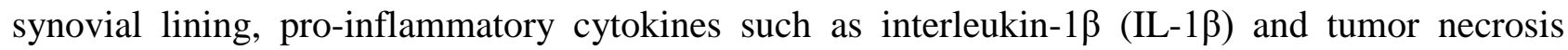


factor- $\alpha(\mathrm{TNF}-\alpha)$ are secreted into the joint. Another soluble factor, galectin-3 (gal-3), is markedly present in synovial tissue during the inflammatory flares. Of note, gal-3 is found in much higher concentrations in RA than in OA synovial tissue [6]. In addition, genetic polymorphisms of galectin-3 may influence the susceptibility to RA, as the galectin-3 gene allele (LGALS3 +292C) is more prevalent in RA patients than in healthy controls [7].

In this mini-review, we will summarize some properties of gal-3 and present available data supporting its pathophysiological role in the different tissues, namely synovium, cartilage and subchondral bone of the joint during arthritis.

\section{Galectin-3: a peculiar member of the galectin family}

Galectins are a family of evolutionary conserved animal lectins which share a high degree of homology in the amino acid sequence of their carbohydrate-recognition domain (CRD). Another common characteristic of these lectins is their affinity for $\beta$-galactosides. To date, 15 galectins have been discovered and classified into three distinct subgroups based on the number and organization of their CRDs (Fig 1).

Prototypic galectins (galectin-1, -2, -5, -7, -10,-11, -13, -14, and -15) contain one CRD and are able to homodimerize. The prototypic galectin-1 is one of the most extensively pro-tumorigenic galectin studied. Its concentration in blood has been demonstrated to correlate with cancer progression in a number of studies. More interestingly, this correlation can be disrupted, for therapeutic purpose, by injection of molecules able to block the CRD activity [8].

Tandem repeat type galectins (galectin-4, -6, -8, -9 and -12) consist of a single polypeptide chain that forms two distinct but homologous CRDs, separated by a linker composed up to 70 amino acids. 
Gal-3, which has been called Mac-2 when first discovered in macrophages, is the unique chimera type in the family of galectins. It was found to be widely distributed in tissues, including gut, brain, kidneys and skeleton [9]. Probably as the best studied member of the galectin family, gal-3 has been shown to amplify inflammatory responses [10] and to play key pathophysiological roles in several biological processes including angiogenesis [11] and development or progression of tumors [12].

\subsection{Galectin-3 synthesis and release}

The gene coding gal-3 has been mapped on human chromosome 14 (14q21-22) and named LGALS3 [13]. The protein is composed of three structural motifs including a short amino-terminal region of 12 amino acids, a collagenase-sensitive sequence rich in G-X-Y tandem repeats that are typical of the collagen supergene family, and a carboxy-terminal moiety containing the globular CRD.

Though gal-3 lacks a signal peptide, it can be secreted via a non-classic pathway called ectocytosis [14]. In this peculiar secretion pathway, cytosolic gal-3 accumulates under the plasma membrane and constitutes aggregates that are first included in evaginating protrusions (blebs) of the membrane. Then, these blebs release the soluble gal-3 into the extracellular space (Fig 2). The secretion of gal-3 depends on cell types and could be facilitated by its interaction with membrane lipids in an energy-independent manner [15]. In non-polarized cells, gal-3 is transported through acidified endosomal compartments bound to vesicular carriers [16]. Whatever the secretion type is, residues 89 to 96 of the $\mathrm{N}$-terminal domain containing proline are crucial [17].

\subsection{Galectin-3 binding properties}

Most functions of gal-3 are supported by its CRD, which binds to glycosylated structures. 
The affinity of galectins increases if galactose is linked to other carbohydrates and glycosylated structures containing $\mathrm{N}$-acetyllactosamine residues are preferential ligands of gal-3 [18]. In addition to this lectinic C-terminal-dependent recognition, gal-3 must multimerize via its $\mathrm{N}$-terminal domain (type-N self-association) to generate biological activities. However, a proteolytic cleavage of gal-3 in the intermediate collagen-like domain increases its binding affinity for the ligands although it does not induce any cell response. Few years ago, a Swedish group demonstrated that whole gal-3 could alternatively multimerize via the CRD, which also resulted in an increased affinity for the ligands. They named this phenomenon, type-C self-association to underline the involvement of the carboxy-terminal domain [19].

To date, identified gal-3 ligands include laminin, fibronectin, hensin, elastin, collagen IV, and integrin $\beta 1$ [20]. A recent report also proposed additional ligands for gal-3 such as prostate-specific antigen, aminopeptidase N, prostatic acid phosphatase, zinc alpha-2-glycoprotein and dipeptidylpeptidase-4 [21].

\subsection{Galectin-3 localization and functions}

Depending on its localization between intracellular and extracellular environments, gal-3 exerts its effects in a carbohydrate-dependent manner but also in an independent way.

Intracellularly, gal-3 shuttles between the nucleus and cytoplasm, being engaged in a variety of cellular processes, such as cell proliferation, differentiation, apoptosis, cytokine secretion, RNA splicing and protein traffic. For instance, intracellular gal-3 can protect $\mathrm{T}$ cells, macrophages and breast carcinoma cells from apoptosis [22,23]. Its anti-apoptotic activity comes from the presence of a NWGR motif within the CRD which is shared with the BH1 domain of Bcl-2 family proteins. In addition, phosphorylation of gal-3 at position $\operatorname{Ser}^{6}$ is also considered essential for the 
anti-apoptotic activity.

Extracellularly, gal-3 has been demonstrated to favour cell-cell or cell-matrix interactions and to induce apoptosis. These characteristics might have relevance for the invasion-metastasis cascade. Indeed, the formation of structures termed lattices, composed of galectin-3 and glycan clusters on the cell surface, modulates tumor cell behavior, such as their adhesion and migration capacity. These lattices also extend the exposure of receptors on the cell surface, therefore affecting the cell response to cytokines and growth factors [24]. Meanwhile, the ability of gal-3 to promote angiogenesis is a vital process for the continuous tumor growth, which provides a pathway for dissemination of malignant cells [25]. Moreover, extracellular gal-3 can induce apoptosis of T cells, activate several lymphoid and myeloid cells including mast cells and T cells, resulting in production of reactive oxygen species (ROS), cell degranulation, and cytokine production [10]. The action of extracellular gal-3 on neutrophils remains controversial, since both apoptotic and anti-apoptotic effects have been reported [26,27].

\section{Pathophysiological role of galectin-3 in synovitis}

The synovial membrane is a specialized mesenchymal tissue lining the spaces of diarthrodial joints. It serves joint function by providing structural support (with a fibrous capsule) and facilitates joint lubrication, through its fibroblast-like cells, by producing the visco-elastic hyaluronate and lubricin, which prevents pathologic deposition of proteins on cartilage surface. Due to the porous organization of its intimal lining and sublining layers, synovium allows diffusion of molecules from the serum to the joint cavity, thus providing nutrients to the avascular cartilage, and contributes to metabolites removal. Synovial macrophages, which are localized in both synovial layers, are considered major players of synovial inflammation due to their prominent role in the production of 
pro-inflammatory cytokines or catabolic products, and ability to activate neighboring synovial fibroblasts. However, other immune cells (mainly $\mathrm{T}$ lymphocytes), which have infiltrated the membrane, can participate too [28], all the more that their migration is facilitated by synovial neovascularization. In both RA and OA, inflamed synovium is characterized by the presence of increased gal-3 levels, which act as a disease amplifier during flares.

\subsection{Arthritic patients and cellular data}

Amongst studies, very few were specifically addressed to OA. However in numerous studies dealing with RA, gal-3 data obtained from OA samples served as control. Compared to OA, the synovium obtained from RA patients express and secrete higher levels of gal-3 [6,29], but one can hypothesize that gal-3 may have, to some extents, similar effects in the joint. In synovitis, the main immune cells found were T cells, macrophages and mast cells, but neutrophils were almost never found [30]. Plasma cells secrete autoantibodies, macrophages and CD4 ${ }^{+}$T-cells activate B cells which themselves activate $\mathrm{CD} 4+\mathrm{T}$ in a feed-back positive mechanism. In addition, $\mathrm{B}$ lymphocytes, as well as macrophages and neutrophils, secrete numerous pro-inflammatory chemokines and cytokines responsible for the continuous leukocyte infiltration and cell activation. Then the chronic aspect of arthritis takes place and results in the persistence of the pannus, permanent activation of immune cells, and secretion of pro-inflammatory soluble mediators by synovium, which lead to degradation of cartilage and bone. In RA, about $39 \%$ of fibroblast-like synoviocytes are cartilage adhering cells and are producing gal-3 in the synovium [31]. Reciprocally, gal-3 also contributes to the activation of RA synovial fibroblasts [6]. Thus, gal-3 over-expression leads the activated synovial fibroblasts and synovial macrophages to be less sensitive to apoptosis, thus favouring their proliferation and the invasion of cartilage by binding to 
the cartilage oligomeric matrix protein (COMP), an important component of hyaline cartilage. RA synovial fibroblasts adhere to COMP with a higher efficacy than OA synovial fibroblasts [31]. In turn, this adhesion stimulates the intracellular expression of gal-3, thus generating a positive feed-back loop, which may be involved in the aggressiveness of synovium. Filer and colleagues [32] showed in vitro that RA synovial fibroblasts, when stimulated with exogenous recombinant gal-3, secreted pro-inflammatory cytokines (IL-6, TNF- $\alpha$ ) and chemokines (CCL2, CCL3, CCL5, CXCL8) through several Mitogen Activated Protein Kinase pathways such as p38, JNK and ERK1/2. All these mediators can induce their own synthesis by activating both synovial fibroblasts and macrophages, yet generating an amplification loop of inflammation. A more recent report demonstrated that LPS-induced IL-6 secretion via TLR receptors is down-regulated in the presence of gal-3 siRNA in human synovial fibroblasts. However, gal-3 siRNAs does not affect MMP3 and CCL5 secretion [33]. In clinical situation, a series of evidence have shown that gal-3 secretion is elevated at the sites of joint destruction, in both RA and OA [6,29,31], and this has been confirmed recently for juvenile RA [34]. The range of gal-3 concentration estimated in the synovial fluid depends on studies. Ohshima et al. found that gal-3 concentration in synovial fluid ranged from 30 to $60 \mathrm{ng} / \mathrm{ml}$ in controls, from 50 to $120 \mathrm{ng} / \mathrm{ml}$ in OA patients and from 130 to $300 \mathrm{ng} / \mathrm{ml}$ in RA patients [6]. In a Turkish study, gal-3 levels in synovial fluid was $3 \mathrm{ng} / \mathrm{ml}$ in controls and $14 \mathrm{ng} / \mathrm{ml}$ in OA patients [29]. Finally in the study dealing with JRA, the gal-3 concentration in synovial fluid ranges from 30 to $70 \mathrm{ng} / \mathrm{ml}$ for patients [34]. Overall, this reinforces the amplifying role of gal-3 in synovial inflammation.

\subsection{Lessons from animal models}

Wang et al. demonstrated that gal-3 was over-expressed in the synovium of 
collagen-induced arthritic (CIA) rats, compared to healthy ones [35], suggesting that this protein could contribute to the pathogenesis of CIA. This group further demonstrated the causative role of gal-3 by inhibiting its expression with RNA interference technology (shGal-3) before arthritis induction [35]. The results show that rats treated with the shGal-3 construct had less severe arthritis assessed by clinical, radiological and histological scoring than untreated ones. Indeed, joints of shGal-3 rats had a more moderate leukocyte infiltration, a decreased number of $\mathrm{T}$ cells, a smaller synovial hyperplasia and a lower density of synovial micro-vessels. Of note, the decreased number of $\mathrm{T}$ cells may be explained by the fact that the depletion of intracellular gal-3 causes apoptosis of these cells. Nonetheless, the pivotal contribution of gal-3 to synovitis has been elegantly demonstrated with gal-3 deficient mice. In gal-3 knock-out $(\mathrm{KO})$ mice, antigen-induced arthritis (AIA) was less severe than in wild-type (WT) mice [36], as demonstrated by a reduced synovial hyperplasia and number of T cells, and lower serum levels of pro-inflammatory cytokines (IL-6, IL-17, TNF- $\alpha$ ). Very importantly, when gal-3 rescue was performed in gal-3 KO mice by intraperitoneal injection of exogenous recombinant gal-3, the severity of joint lesions and the levels of pro-inflammatory cytokines became similar to those observed in WT mice. Taken together, these results show that invalidation of gal-3 improves the symptoms of experimental arthritis, suggesting that this lectin is involved in the pathophysiology of RA. Moreover, an intra-articular injection of gal-3 induced swelling and OA-like lesions in naive mice [37], further highlighting that the presence of gal-3 into the joints correlates with synovial inflammation and cartilage/bone degradation.

\section{Pathophysiological role of galectin-3 in cartilage degradation}

Normal articular cartilage is composed of a dense extracellular matrix (ECM) with sparse 
distribution of chondrocytes, which are responsible for the production of ECM components. Amongst major ECM molecules, aggrecan endows the cartilage with compressive resistant and shock absorbing capability, whereas collagens, mainly represented by the type II, accounts for the tensile property of hyaline cartilage. Along with a variety of glycoproteins and non-collagenous proteins, aggrecan and collagens form a matrix framework to protect the chondrocytes from the harmful effects of excessive loading. Chondrocytes are quiescent in normal adult cartilage, but this condition is disrupted when arthritis occurs. To compensate for the missing ECM, these cells increase the synthesis of matrix macromolecules by producing growth factors. However, when they failed to restore the tissue, chondrocytes decrease their anabolic metabolism and produce degrading enzymes. The main source of degrading enzymes contributing to cartilage lesions differs between RA and OA. The former takes the inflammatory synovium as the principle source while the latter puts quickly the stimulated chondrocytes on a prominent position. These enzymes including collagenases and aggrecanases belong to the MMP and the ADAMTS (A Disintegrin And Metalloproteinase with Thrombospondin Motifs) families. The matrix degradation in early OA may be due to proteoglycan loss induced by MMP3 and ADAMTS5 or 4, depending on species. Afterwards, the collagenase activity will increase, especially MMP13, which is highly efficient in degrading type II collagen. Once this collagen network is degraded, the cartilage reaches a pathological step that cannot be reversed.

\subsection{Intracellular galectin-3 and chondrocyte data}

Colnot et al identified the expression of gal-3 in differentiated chondrocytes with a higher concentration in the cytoplasm of mature and early hypertrophic chondrocytes than in the late hypertrophic ones [38]. In the late stage of long bone development, gal-3 KO mouse embryos have 
a decreased number of hypertrophic chondrocytes accompanied with an increased number of empty lacunae and condensed chondrocytes at the chondro-vascular junction. The up-regulation of gal-3 in hypertrophic cartilage has also been reported both in chicken [39] and during the first trimester of human embryogenesis [9]. In cultured chondrocytes from OA patients, an intracellular induction of gal-3 has been reported, as well as an increase of its secretion [40]. These variations of the intracellular content of gal-3 indicate its contribution to chondrocyte physiology. For example, it was suggested that gal-3 could be involved both in the chondrocyte death and the vascular invasion coupling process during endochondral ossification [41]. The increase of gal-3 expression in chondrocytes in both prehypertrophic stage and during osteoarthritis might be related to the necessity to maintain suffering chondrocytes in a survival state as long as possible. Indeed, in both conditions, it has been reported that the level of ATP was down regulated $[42,43]$, indicating an impaired metabolic status. The contribution of intracellular gal-3 to chondrocyte survival could be explained, at least in part, by considering that chondrocytes impairment, secondary to mitochondrial dysfunction, contributes to the initiation and progression of OA, and by the fact that gal-3 prevents mitochondrial damage and cytochrome c release [44]. Consistently with this proposal, the cartilage of gal-3 KO mice has a higher degenerative score than that of WT mice [45].

\subsection{Extracellular galectin-3 and chondrocyte data}

At different concentrations, extracellular gal-3 induces MMP3 and ADAMTS5 expression in chondrocytes, which are two main enzymes responsible for proteoglycan degradation in cartilage. When gal-3 was injected into mouse knees, swelling and OA-like lesions occurred [37]. Two of the well-known gal-3 ligands, namely fibronectin and integrin $\beta 1$, are also found in increased amount in OA cartilage and one can hypothesize that their recognition by gal-3 may stimulate chondrocytes 
towards an enhanced ECM degrading activity. Moreover, the altered glycophenotype of OA chondrocytes was shown to correlate with progressing cartilage degeneration in areas where galectins are present to translate the sugar code modifications into biological functions [46]. The authors showed that gal-3 binds preferentially to chondrons and pannus-like tissue, while galectin-1 takes inter-territorial matrix of cartilage and blood vessels as main binding sites [46].

\section{Pathophysiological role of galectin-3 in subchondral bone remodeling}

The subchondral bone (SCB) plate is the plate underneath cartilage. The line separating calcified cartilage from non-calcified cartilage, called tidemark, is crossed by type II collagen [47], while cement line is the border between cartilage and SCB. SCB is particularly highly vascularized. The high rates of bone blood flow are associated with increased bone remodeling. The architecture of SCB adapts to the mechanical alterations transmitted to the joints, as illustrated by the elevated thickness, vascularity and perforations found in greater stress regions [47]. SCB is specialized in load bearing, as it attenuates loadings with an efficiency of about 10 times better than cartilage. The deep layer of non-calcified cartilage, the tidemark, calcified cartilage, the cement line and SCB construct an integrated complex called osteochondral junction. Though the complex is vital for normal joint function, it also provides the convenience to disease progression such as OA. During OA, upward migration of SCB, bone sclerosis, osteophyte formation, SCB cysts and bone marrow oedema-like lesions characterize the SCB alterations [48]. In addition, the increased vascularization and the development of microcracks in the bone matrix reinforce the interaction between cartilage and SCB, as more penetrating channels allow for the exchange areas [49]. As a consequence, factors secreted by one of other tissue will follow these channels and modify the biological behavior of the non-secreting ones. For instance, IGF-1 and TGF- $\beta$, two growth factors for SCB, are secreted 
in high amounts by deteriorated cartilage, and will partly account for the bone sclerosis. On the other hand, the degradation of cartilage exposes SCB to more loading pressure. However, as its capacity to respond to mechanical stress reduces due to its alterations in $\mathrm{OA}, \mathrm{SCB}$ will in turn make the articular cartilage to suffer from increased loading pressure, which will result in more severe cartilage degradation.

\subsection{Galectin-3 and osteoblast data}

Expression of gal-3 in osteoblastic cells has been proven and confirmed by a variety of studies [50,51]. Runx2, a transcription factor with a key role in skeletal development, stimulates gal-3 expression in a preosteoblastic murine cell line [51]. Interestingly, dynamic mechanical loading leads to the induction of bone density and strength, and promotes osteoblast proliferation and differentiation, as well as matrix production by activating Runx2. This suggests that intracellular gal-3 may play an important role in the osteoblast physiology.

It was recently reported that gal-3 inhibits human osteoblast differentiation through its binding to Notch 1, which leads to the activation of Notch signaling [52]. Gal-3 was also identified as one of the two principal receptors of advanced glycation end products (AGEs). Mercer et al have demonstrated that AGEs induce the expression and secretion of gal-3 in osteoblast-like cells, accompanied with cell apoptosis [53]. The apoptosis may indirectly proceed by AGEs through increasing oxidative stress, or via stimulating pro-apoptotic cytokines expression. However, since the accumulation of AGEs, which increases stiffness of collagen network in bone, is a characteristic of the tissue in aged people, their binding to gal-3 probably have significant consequences on osteoblast metabolism and thus, on bone turnover [53]. Finally, gal-3 injection into the mouse knees has been demonstrated to play a deleterious role on osteoblasts metabolism, as it inhibits 
osteoblastic markers expression such as osteocalcin [37]. Such impairment of the osteoblast phenotype could participate in the abnormal mineralization reported during inflammatory phases of OA.

\subsection{Galectin-3 and osteoclast data}

Two pioneering studies showed that pre-osteoclasts express gal-3 [54]. In addition, Niida et al demonstrated, by using electron microscopy, that gal-3 is distributed on the plasma membrane of pre-osteoclasts, as well as in their cytoplasm and periphery of nucleus, and also in the extracellular matrix between the cells and bone indicating that gal-3 is secreted [54]. Using the adjuvant-induced arthritis rat model, which is characterized by severe bone destruction, Li et al demonstrated that gal-3 suppresses significantly bone destruction and osteoclast recruitment [55]. They also showed that in vitro, extracellular gal-3 inhibited osteoclastogenesis and reduced dentine resorption by mature osteoclasts. The full length gal-3 was also previously identified as an inhibitor of osteoclastogenesis in MMP9 deficient mice. Indeed this metalloproteinase cleaves extracellular gal-3 in the collagen-like domain, which prevents its multimerization and removes the osteoclastogenesis inhibition [56]. More recently, osteolytic metastases of breast cancer were correlated to the presence of MMP13 [57], another metalloproteinase able to cleave gal-3 [40]. The authors demonstrated that MMP13 and potentially MMP9 promoted osteoclastogenesis by cleaving gal-3 [57].

\section{Conclusion and perspectives}

In summary, gal-3 is mainly expressed and secreted by the inflamed synovium which accounts for its higher synovial fluid levels in RA than in OA. In arthritis conditions, synovial tissue, cartilage and SCB are exposed to elevated gal-3 levels, which may play a causative role in the initiation 
and/or progression of the diseases due to its pro-inflammatory and pro-catabolic potencies (Table 1). Though some progresses have been made in the past decades, the definite role of gal-3 in RA and OA has unfortunately not been fully clarified due to the difficulty to consider its intracellular effects in animal models. However, there are already cellular and preclinical evidences supporting that gal-3 blockade could be beneficial in arthritis, opening some avenues for the development of gal-3 inhibitors.

\section{Acknowledgments}

This work was supported by the Osteoarthritis Chair of Excellence (CNRS/Universite de Lorraine) and in part by le comité régional de la Fondation Médicale pour la Recherche and la Région Lorraine to P Reboul. Y. HU was an award recipient from the government of Republic of China. M. Yéléhé-Okouma was an award recipient from l'Agence Nationale des Bourses du Gabon and l’Agence Régionale de Santé Lorraine. 


\section{References}

1. Guillemin F, Rat AC, Mazieres B, et al. Prevalence of symptomatic hip and knee osteoarthritis: a two-phase population-based survey. Osteoarthritis Cartilage 2011;19:1314-22.

2. Guillemin F, Saraux A, Guggenbuhl P, et al. Prevalence of rheumatoid arthritis in France: 2001. Ann Rheum Dis 2005;64:1427-30.

3. Sellam J, Berenbaum F. The role of synovitis in pathophysiology and clinical symptoms of osteoarthritis. Nat Rev Rheumatol 2010;6:625-35.

4. Fernandez-Madrid F, Karvonen RL, Teitge RA, et al. Synovial thickening detected by MR imaging in osteoarthritis of the knee confirmed by biopsy as synovitis. Magn Reson Imaging 1995;13:177-83.

5. Rosado-de-Castro PH, Lopes de Souza SA, Alexandre D, et al. Rheumatoid arthritis: Nuclear Medicine state-of-the-art imaging. World J Orthop 2014;5:312-18.

6. Ohshima S, Kuchen S, Seemayer CA, et al. Galectin 3 and its binding protein in rheumatoid arthritis. Arthritis Rheum 2003;48:2788-95.

7. Hu CY, Chang SK, Wu CS, et al. Galectin-3 gene (LGALS3) +292C allele is a genetic predisposition factor for rheumatoid arthritis in Taiwan. Clin Rheumatol 2011;30:1227-33.

8. Ito K, Stannard K, Gabutero E, et al. Galectin-1 as a potent target for cancer therapy: role in the tumor microenvironment. Cancer Metastasis Rev 2012;31:763-78.

9. van den Brule FA, Fernandez PL, Buicu C, et al. Differential expression of galectin-1 and galectin-3 during first trimester human embryogenesis. Dev Dyn 1997;209:399-405.

10. Sundblad V, Croci DO, Rabinovich GA. Regulated expression of galectin-3, a multifunctional glycan-binding protein, in haematopoietic and non-haematopoietic tissues. Histol Histopathol 2011;26:247-65.

11. Markowska AI, Liu FT, Panjwani N. Galectin-3 is an important mediator of VEGF- and bFGF-mediated angiogenic response. J Exp Med 2010;207:1981-93.

12. Liu FT, Rabinovich GA. Galectins as modulators of tumour progression. Nat Rev Cancer 2005;5:29-41.

13. Raimond J, Zimonjic DB, Mignon C, et al. Mapping of the galectin-3 gene (LGALS3) to human chromosome 14 at region 14q21-22. Mamm Genome 1997;8:706-07.

14. Lepur A, Carlsson MC, Novak R, et al. Galectin-3 endocytosis by carbohydrate independent and dependent pathways in different macrophage like cell types. Biochim Biophys Acta 2012;1820:804-18. 
15. Hughes RC. Secretion of the galectin family of mammalian carbohydrate-binding proteins. Biochim Biophys Acta 1999;1473:172-85.

16. Schneider D, Greb C, Koch A, et al. Trafficking of galectin-3 through endosomal organelles of polarized and non-polarized cells. Eur J Cell Biol 2010;89:788-98.

17. Menon S, Kang CM, Beningo KA. Galectin-3 secretion and tyrosine phosphorylation is dependent on the calpain small subunit, Calpain 4. Biochem Biophys Res Commun 2011;410:91-6.

18. Sato S, Hughes RC. Binding specificity of a baby hamster kidney lectin for H type I and II chains, polylactosamine glycans, and appropriately glycosylated forms of laminin and fibronectin. J Biol Chem 1992;267:6983-90.

19. Lepur A, Salomonsson E, Nilsson UJ, et al. Ligand induced galectin-3 protein self-association. J Biol Chem 2012;287:21751-56.

20. Fortuna-Costa A, Gomes AM, Kozlowski EO, et al. Extracellular galectin-3 in tumor progression and metastasis. Front Oncol 2014;4:138.

21. Kovak MR, Saraswati S, Goddard SD, et al. Proteomic identification of galectin-3 binding ligands and characterization of galectin-3 proteolytic cleavage in human prostasomes. Andrology 2013;1:682-91.

22. Hoyer KK, Pang M, Gui D, et al. An anti-apoptotic role for galectin-3 in diffuse large B-cell lymphomas. Am J Pathol 2004;164:893-902.

23. Dumic J, Dabelic S, Flogel M. Galectin-3: an open-ended story. Biochim Biophys Acta 2006;1760:616-35.

24. Rabinovich GA, Toscano MA, Jackson SS, et al. Functions of cell surface galectin-glycoprotein lattices. Curr Opin Struct Biol 2007;17:513-20.

25. Blidner AG, Rabinovich GA. 'Sweetening' pregnancy: galectins at the fetomaternal interface. Am J Reprod Immunol 2013;69:369-82.

26. Fernandez GC, Ilarregui JM, Rubel CJ, et al. Galectin-3 and soluble fibrinogen act in concert to modulate neutrophil activation and survival: involvement of alternative MAPK pathways. Glycobiology 2005;15:519-27.

27. Farnworth SL, Henderson NC, Mackinnon AC, et al. Galectin-3 reduces the severity of pneumococcal pneumonia by augmenting neutrophil function. Am J Pathol 2008;172:395-405.

28. Scanzello CR, Goldring SR. The role of synovitis in osteoarthritis pathogenesis. Bone 2012;51:249-57. 
29. Alturfan AA, Eralp L, Emekli N. Investigation of inflammatory and hemostatic parameters in female patients undergoing total knee arthroplasty surgery. Inflammation 2008;31:414-21.

30. de Lange-Brokaar BJ, Ioan-Facsinay A, van Osch GJ, et al. Synovial inflammation, immune cells and their cytokines in osteoarthritis: a review. Osteoarthritis Cartilage 2012;20:1484-99.

31. Neidhart M, Zaucke F, von Knoch R, et al. Galectin-3 is induced in rheumatoid arthritis synovial fibroblasts after adhesion to cartilage oligomeric matrix protein. Ann Rheum Dis 2005;64:419-24.

32. Filer A, Bik M, Parsonage GN, et al. Galectin 3 induces a distinctive pattern of cytokine and chemokine production in rheumatoid synovial fibroblasts via selective signaling pathways. Arthritis Rheum 2009;60:1604-14.

33. Arad U, Madar-Balakirski N, Angel-Korman A, et al. Galectin-3 is a sensor-regulator of toll-like receptor pathways in synovial fibroblasts. Cytokine 2015;73:30-5.

34. Ezzat MH, El-Gammasy TM, Shaheen KY, et al. Elevated production of galectin-3 is correlated with juvenile idiopathic arthritis disease activity, severity, and progression. Int $\mathbf{J}$ Rheum Dis 2011;14:345-52.

35. Wang CR, Shiau AL, Chen SY, et al. Intra-articular lentivirus-mediated delivery of galectin-3 shRNA and galectin-1 gene ameliorates collagen-induced arthritis. Gene Ther 2010;17:1225-33.

36. Forsman H, Islander U, Andreasson E, et al. Galectin 3 aggravates joint inflammation and destruction in antigen-induced arthritis. Arthritis Rheum 2011;63:445-54.

37. Janelle-Montcalm A, Boileau C, Poirier F, et al. Extracellular localization of galectin-3 has a deleterious role in joint tissues. Arthritis Res Ther 2007;9:R20.

38. Colnot C, Sidhu SS, Poirier F, et al. Cellular and subcellular distribution of galectin-3 in the epiphyseal cartilage and bone of fetal and neonatal mice. Cell Mol Biol (Noisy-le-grand) 1999;45:1191-202.

39. Nurminskaya M, Linsenmayer TF. Identification and characterization of up-regulated genes during chondrocyte hypertrophy. Dev Dyn 1996;206:260-71.

40. Guevremont M, Martel-Pelletier J, Boileau C, et al. Galectin-3 surface expression on human adult chondrocytes: a potential substrate for collagenase-3. Ann Rheum Dis 2004;63:636-43.

41. Colnot C, Sidhu SS, Balmain N, et al. Uncoupling of chondrocyte death and vascular invasion in mouse galectin 3 null mutant bones. Dev Biol 2001;229:203-14. 
42. Matsumoto H, DeBolt K, Shapiro IM. Adenine, guanine, and inosine nucleotides of chick growth cartilage: relationship between energy status and the mineralization process. J Bone Miner Res 1988;3:347-52.

43. Johnson K, Svensson CI, Etten DV, et al. Mediation of spontaneous knee osteoarthritis by progressive chondrocyte ATP depletion in Hartley guinea pigs. Arthritis Rheum 2004;50:1216-25.

44. Matarrese P, Tinari N, Semeraroa ML, et al. Galectin-3 overexpression protects from cell damage and death by influencing mitochondrial homeostasis. FEBS Lett 2000;473:311-15.

45. Boileau C, Poirier F, Pelletier JP, et al. Intracellular localization of Galectin-3 has a protective role. Ann Rheum Dis 2008;67:175-81.

46. Toegel S, Bieder D, Andre S, et al. Glycophenotyping of osteoarthritic cartilage and chondrocytes by RT-qPCR, mass spectrometry, histochemistry with plant/human lectins and lectin localization with a glycoprotein. Arthritis Res Ther 2013;15:R147.

47. Madry H, van Dijk CN, Mueller-Gerbl M. The basic science of the subchondral bone. Knee Surg Sports Traumatol Arthrosc 2010;18:419-33.

48. Orth P, Cucchiarini M, Kohn D, et al. Alterations of the subchondral bone in osteochondral repair--translational data and clinical evidence. Eur Cell Mater 2013;25:299-316; discussion 14-6.

49. Burr DB, Radin EL. Microfractures and microcracks in subchondral bone: are they relevant to osteoarthrosis? Rheum Dis Clin North Am 2003;29:675-85.

50. Aubin JE, Gupta AK, Bhargava U, et al. Expression and regulation of galectin 3 in rat osteoblastic cells. J Cell Physiol 1996;169:468-80.

51. Stock M, Schafer H, Stricker S, et al. Expression of galectin-3 in skeletal tissues is controlled by Runx2. J Biol Chem 2003;278:17360-67.

52. Nakajima K, Kho DH, Yanagawa T, et al. Galectin-3 inhibits osteoblast differentiation through notch signaling. Neoplasia 2014;16:939-49.

53. Mercer N, Ahmed H, Etcheverry SB, et al. Regulation of advanced glycation end product (AGE) receptors and apoptosis by AGEs in osteoblast-like cells. Mol Cell Biochem 2007;306:87-94.

54. Niida S, Amizuka N, Hara F, et al. Expression of Mac-2 antigen in the preosteoclast and osteoclast identified in the op/op mouse injected with macrophage colony-stimulating factor. J Bone Miner Res 1994;9:873-81.

55. Li YJ, Kukita A, Teramachi J, et al. A possible suppressive role of galectin-3 in upregulated 
osteoclastogenesis accompanying adjuvant-induced arthritis in rats. Lab Invest 2009;89:26-37.

56. Ortega N, Behonick DJ, Colnot C, et al. Galectin-3 is a downstream regulator of matrix metalloproteinase-9 function during endochondral bone formation. Mol Biol Cell 2005;16:3028-39.

57. Pivetta E, Scapolan M, Pecolo M, et al. MMP-13 stimulates osteoclast differentiation and activation in tumour breast bone metastases. Breast Cancer Res 2011;13:R105. 


\section{Legends of figures}

Figure 1: The structure and classification of different members of the galectin family. (adapted from Blidner AG et al. 2013 and Lepur A et al. 2012). Galectins are classified into three distinct subgroups based on the number and organization of their Carbohydrate Recognition Domain. a) Prototypic galectins (galectin-1, -2, -5, -7, -10, -11, -13, -14, and -15) contain one CRD able to homodimerize. b) Tandem repeat type galectins (galectin-4, -6, -8, -9 and -12) consist of a single polypeptide containing two distinct but homologous CRDs, separated by a linker of 70 amino acids. c) Gal-3 is the unique chimera type. In addition to this lectinic dependent recognition, gal-3 can multimerize via its N-terminal domain (type-N self-association) or its CRD (type-C self-association).

Figure 2: The details of galectin-3 secretion. To be secreted, galectin-3 accumulates in the cytoplasm underlying plasma membrane domains. These aggregates evaginate and merge with the plasma membrane to form a protrusion that pinch off to release extracellular vesicles from which soluble lectin is released. (Adapted from Hughes RC et al. 1999) 


\section{Figure 1}

(a) Prototypic galectins (galectin-1, $-2,-5,-7,-10,-11,-13,-14,-15$ )

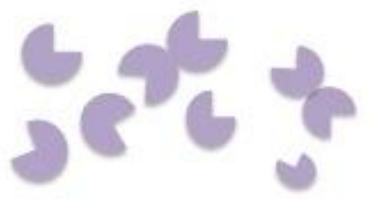

(b) Tandem repeat type galectins (galectin-4, -6, -8, -9, -12)

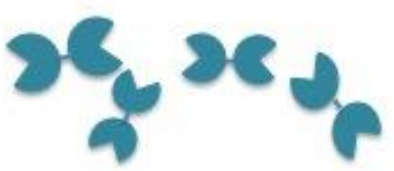

(c) Chimera galectin (galectin-3)

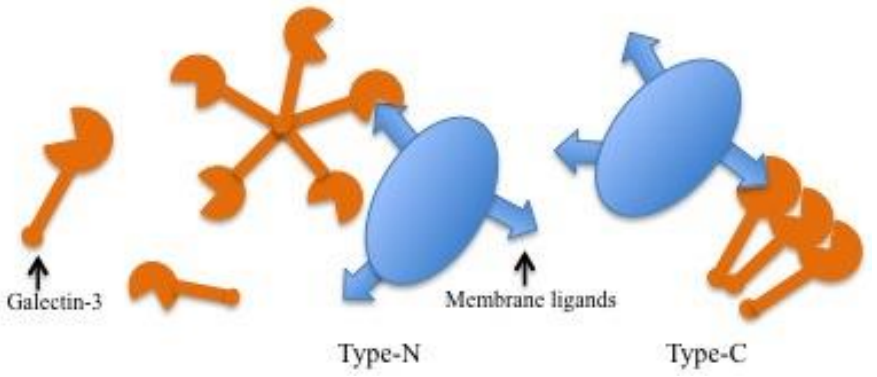

Figure 2

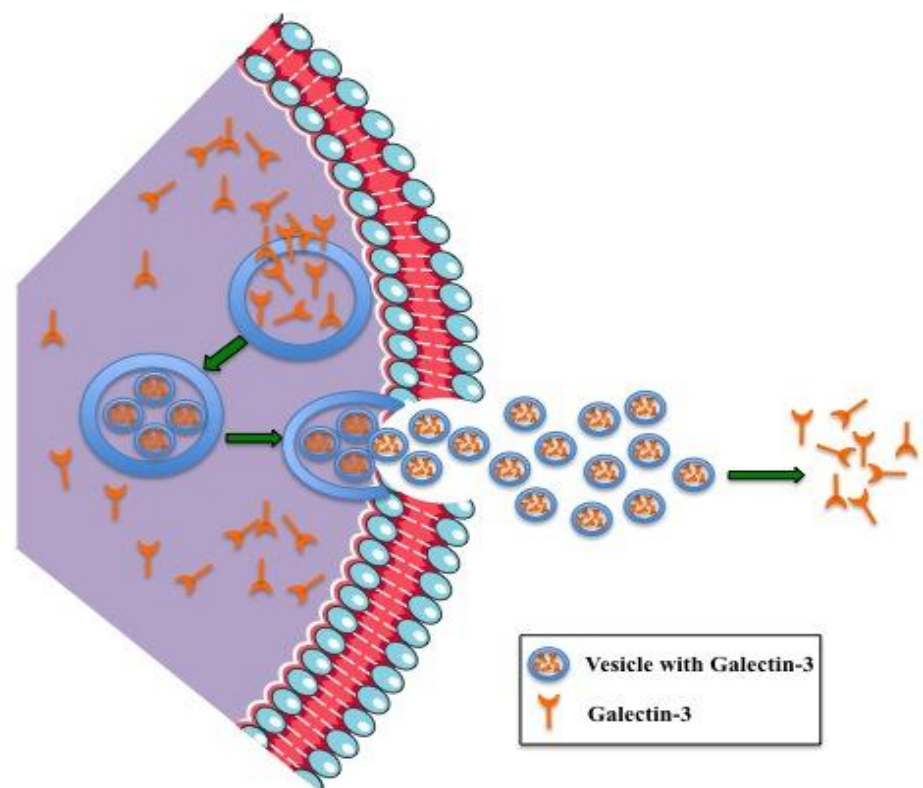


Table 1: Roles of galectin-3 in joint tissues

\begin{tabular}{|c|c|}
\hline Extracellular roles of galectin-3 roles on: & References \\
\hline \multicolumn{2}{|l|}{ In Synovium, gal-3 } \\
\hline Is secreted by $\mathrm{OA}$ and RA synovial fibroblasts & 6 \\
\hline Stimulates the growth of OA and RA synovial fibroblast cultures & 6 \\
\hline Induces pro-inflammatory cytokines and chemokines in RA synovial fibroblasts & 32 \\
\hline Secretion is elevated at the sites of joint destruction & 6,34 \\
\hline \multicolumn{2}{|l|}{ Contributes to the pathogenesis of the collagen-induced arthritis and } \\
\hline antigen-induced arthritis models & 35,36 \\
\hline Induces OA-like lesion when injected into mouse knees & 37 \\
\hline \multicolumn{2}{|l|}{ In Cartilage, gal-3 } \\
\hline Induces MMPs and ADAMTS in chondrocytes & 37 \\
\hline Is associated with progressing cartilage degeneration & 46 \\
\hline \multicolumn{2}{|l|}{ In Subchondral bone, gal-3 } \\
\hline Inhibits osteoblast differentiation & 37,52 \\
\hline Suppresses bone destruction, osteoclast recruitment and inhibits osteoclastogenesis & 55,56 \\
\hline \multicolumn{2}{|l|}{ Intracellular roles of galectin-3 roles on: } \\
\hline \multicolumn{2}{|l|}{ In Synovium, gal-3 } \\
\hline Is increased in RA synovio-fibroblasts in the presence of COMP & 31 \\
\hline \multicolumn{2}{|l|}{ In Cartilage, gal-3 } \\
\hline Is expressed in hypertrophic chondrocyte and OA chondrocytes & $38-40$ \\
\hline Prevents chondrocytes from death cell & 41,45 \\
\hline Deficient mice have OA-like lesions & 45 \\
\hline \multicolumn{2}{|l|}{ In Subchondral bone, gal-3 } \\
\hline Is increased during osteoblast differentiation under the control of RUNX2 & 50,51 \\
\hline
\end{tabular}

